\title{
An Occlusive Left Main Spontaneous Coronary Artery Dissection
}

\author{
Mehdi Bamous*, Jacques Robin, Younes Moutakiallah, Fouad Nya, Olivier Metton, Jean Ninet
}

Department of Cardiothoracic Surgery, Luis Pradel Hospital, University of Claude Bernard 1, Lyon, France.

Email: *mehdibamous@hotmail.com

Received January $14^{\text {th }}, 2013$; revised February $15^{\text {th }}, 2013$; accepted February $23^{\text {rd }}, 2013$

\begin{abstract}
Spontaneous coronary artery dissection (SCAD) should be strongly suspected in young women with few or no traditional risk factors for atherosclerosis. Prompt diagnosis and treatment improve survival. The most appropriate strategy for managing SCAD is still controversial due to the heterogeneity of this population. We describe a case of spontaneous left main coronary artery (LMCA) dissection in a young women that was successfully managed by percutaneous coronary intervention (PCI) followed by coronary artery bypass grafting (CABG), this case illustrates the utility of coronary computed tomography (CT) and magnetic resonance imagery (MRI) in assessing complex coronary dissection, thereby helping to determine the best timing for surgery.
\end{abstract}

Keywords: Coronary Dissection; Coronary Artery Imaging; MRI; Computed Tomography; CABG; Arterial Grafts

\section{Introduction}

Coronary artery dissection is termed spontaneous upon exclusion of secondary causes such as cardiac catherization, chest trauma, aortic root dissection and cardiac surgery. The diagnosis is usually made by urgent coronary angiography. Therapeutic options include medical therapy, PCI and surgery. Because dissection may recur, on the coronary vessels or in other vascular bed, extended follow-up should be considered.

We present a case of young women who presented with an acute coronary syndrome (ACS) due to an occlusive SCAD involving left main stem. CT coronary angiography and cardiac MRI were used to document the persistence of the dissection and to guide the decision to proceed with surgical revascularisation.

\section{Case Report}

A previously healthy 34-year-old female presented with sudden onset of severe chest pain at rest. Her only cardiac risk factor was smoking (8 pack-year).she has no history of connective tissue disease, oral contraceptive or illicit drug use and she wasn't pregnant. On admission, her heart rate was 85 beats/min and blood pressure was 110/78 mmHg. Cardiopulmonary examination didn't reveal any abnormalities. The electrocardiogram showed normal sinus rhythm with widespread T-wave inversion. Cardiac biomarkers were elevated with a creatine kinase

*Corresponding author. of $600 \mu \mathrm{g} / \mathrm{l}$ (normal range 0 - 165) and cardiac troponin I of $9 \mu \mathrm{g} / \mathrm{l}$ (normal range 0.01 - 0.05). Echocardiography showed an apical hypokinesia with ejection fraction of $55 \%$. The diagnosis of ACS was made and the patient was taken to coronary angiography which revealed an occlusive dissection of left main stem and left anterior descending artery (LAD) with thrombolysis in myocardial infarction (TIMI) 0 flow (Figure 1). In view of this critical setting of "no flow" SCAD, urgent balloon angioplasty was performed which permitted restoration of antegrade flow in LAD with TIMI 3 flow (Figure 2). The dissection flap was starting from the left coronary ostium and progressing to the LAD and circumflex (Cx) arteries, the right coronary artery (RCA) was normal. Based on the vessels morphology and restoration of flow in LMCA, we decide to manage this patient conservatively with close follow-up at the intensive care unit (ICU).The patient was treated with aspirin, clopidogrel, bisoprolol, ramipril and unfractionned heparin for ACS. During hospital stay, she remained asymptomatic, a 64 slice CT scanner and cardiac MRI were used to monitor flow in LMCA and to determine if the dissection flap was persistent or had spontaneously healed. Four weeks after her admission, the study confirmed a persisting LMCA dissection with a spiral extension along the LAD artery (Figures 3 and 4). She was subsequently referred for CABG. At operation, the false and true lumens were carefully identified for conduit anastomosis. On extracorporeal circulation, right internal mammary artery was 


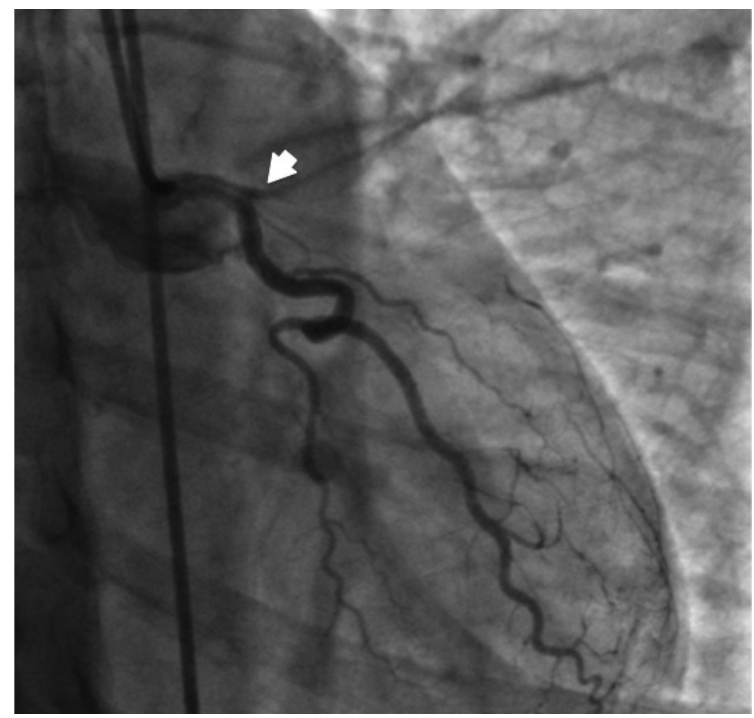

Figure 1. Coronary angiogram showing obstruction of the left main stem (arrow) with occlusion of the left anterior descending and the first obtuse marginal arteries.

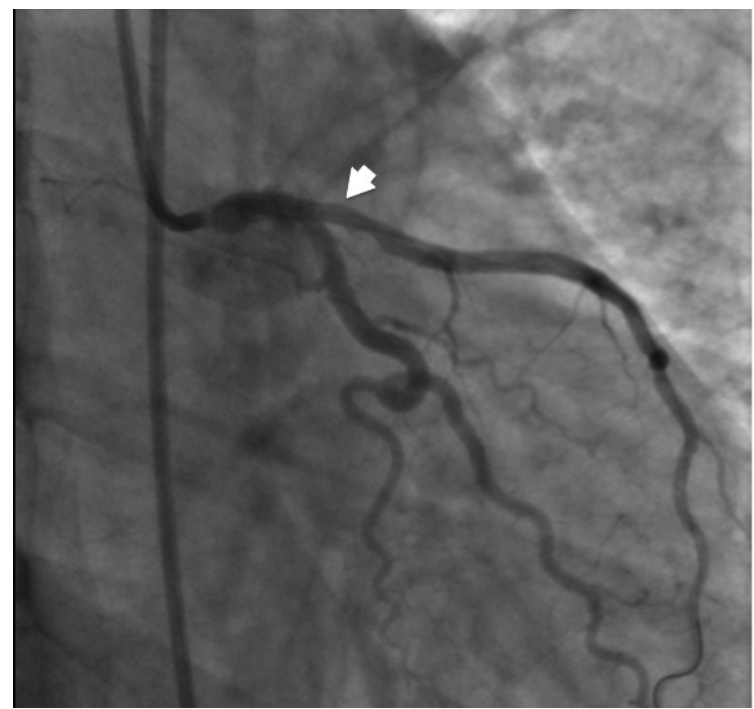

Figure 2. Coronary angiogram demonstrating an antegrade flow in the left main and left anterior descending arteries with the dissection flap (arrow).

used as the conduit for LAD while left internal mammary artery was anastomosed to the first obtuse marginal artery. She had an uneventful recovery before being discharged from the hospital one week post-operatively. At one year follow-up, her cardiac exercise testing was normal and TTE demonstrated normal ventricular wall motion with an ejection fraction of $60 \%$.

\section{Discussion}

Since the first description of SCAD in 1931, as a cause of myocardial infarction, an accurate knowledge of the true prevalence is unknown. However, the incidence is

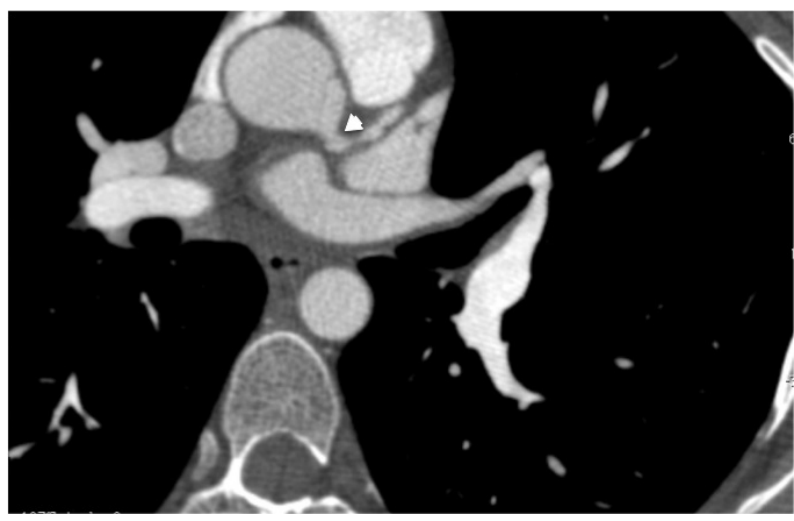

(a)

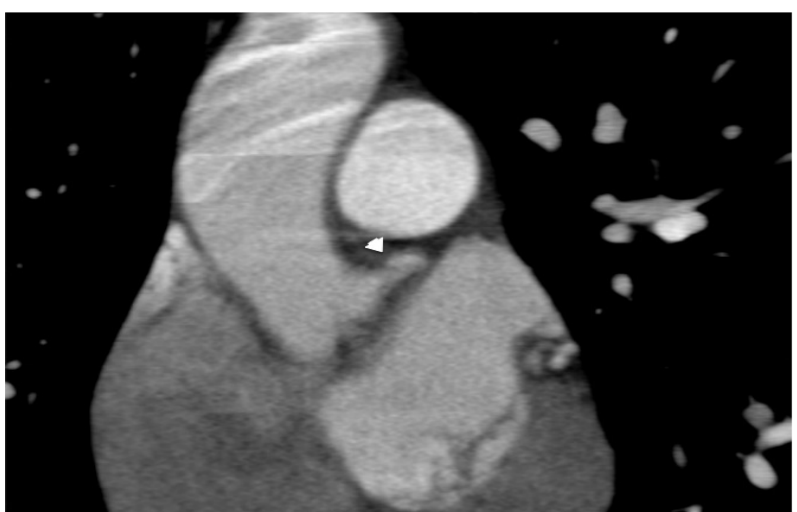

(b)

Figure 3. Coronary computed tomography showing the intimal flap in the left main and the proximal part of the left anterior descending artery (arrow). (a) Axial; (b) Coronal images.

estimated between $0.1 \%$ and $0.28 \%$ of all patients with ACS [1]. The pathological mechanism is poorly understood but there are a number of well-described predisposing factors, in particular, female gender during pregnancy or the early post partum period, atherosclerosis and inflammatory conditions affecting the arterial wall such as marfan's syndrome and other collagen disorders.

Recently, some authors have proposed a potential association with oral contraceptive use [2], intensive exercice and illicit drug use [3]. Overall, the LAD is affected in $75 \%$ of cases, the RCA in $20 \%$, the $\mathrm{Cx}$ in $4 \%$ and the LMCA in less than $1 \%$ of cases [3].

The diagnosis of SCAD by selective angiography depends on the visualisation of two lumina separated by radiolecent intimal flap. Intravascular ultrasound (IVUS) can aid in diagnosis particularly when the angiographic intimal flap is not evident [4]. Finally, non-invasive coronary angiography such as computed tomography and MRI have increased recognition of SCAD and may be useful in assessing dissection limited to the medial adventitial layers.

Total obstruction of LAD and first marginal arteries is quite rare condition complicating left main stem SCAD 


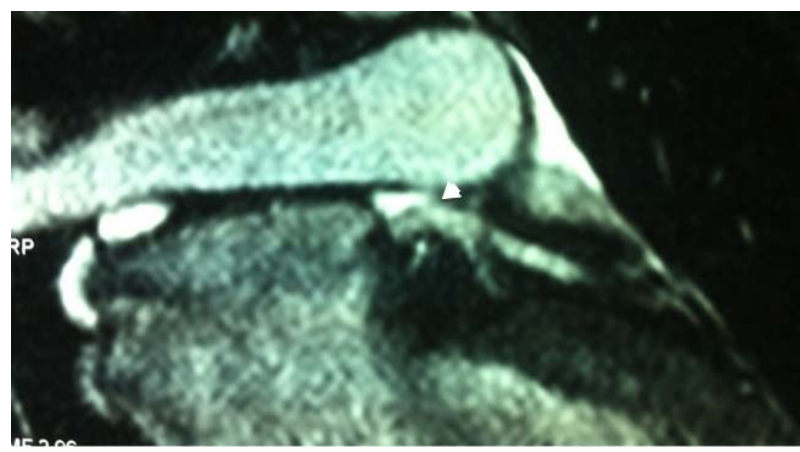

(a)

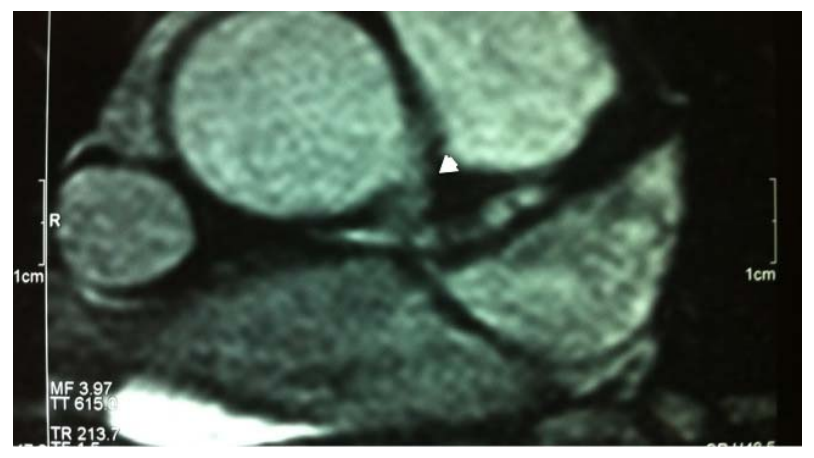

(b)

Figure 4. Cardiac MRI demonstrating a persistent dissecttion flap at the left coronary ostium and left main stem (arrow). (a) Coronal; (b) Axial images.

and no evidence-based data were available to guide decision making in this patient. Because balloon angioplasty has permitted a suitable antegrade flow in the LAD and some SCAD may resolve safely with medical therapy alone [5], we initially elected to defer revascularisation and to monitor the patient closely at the ICU. Then, we chose using cardiac CT and MRI to re-assess her coronary anatomy instead of repeated invasive coronary angiography. Assessment with cardiac CT and MRI revealed high diagnostic accuracy, so we didn't need additional information from any other imaging techniques that could change our clinical decision for this patient.

Revascularization with PCI is not without its difficulties, the true lumen of the vessel may be difficult to wire, particularly when the dissection involves the ostium of the LMCA. In addition, if the entire length of the vessel is stented this will inevitably limit the possibility of performing CABG and may result in a large periprocedural myocardial infarction [6]. A PCI is therefore best limited to patients with localized dissection rather those with extensive one, as our case. CABG can be considered as first-line treatment in multivessel SCAD, new-onset heart failure or left main stem involvement. Even surgery may be challenging, in particular, grafting to the true lumen can be difficult, the arterial wall is often fragile due to the underlying condition predisposing to dissection and when the patient is extremely unstable. Finally, off-pump CABG can be proposed for pregnant patients [7]. In accordance with other rare conditions, the literature is based on case reports and further research is needed to provide prospective data that will help in understanding the processes involved in SCAD therefore, optimizing management of this group of patients.

\section{REFERENCES}

[1] A. Fontanelli, Z. Olivari, L. La Vecchia, et al., "Spontaneous Dissections of Coronary Arteries and Acute Coronary Syndromes: Rationale and Design of the DISCOVERY, a Multicenter Prospective Registry with a CaseControl Group," Journal of Cardiovascular Medicine, Vol. 10, No. 1, 2009, pp. 94-99. doi:10.2459/JCM.0b013e32830f45c7

[2] D. Evangelou, K. P. Letsas, P. Korantzopoulos, I. Antonellis, E. Sioras and F. Kardaras, "Spontaneous Coronary Artery Dissection Associated with Oral Contraceptive Use: A Case Report and Review of the Literature,” International Journal of Cardiology, Vol. 112, No. 3, 2006, pp. 380-382. doi:10.1016/j.ijcard.2005.07.069

[3] M. Q. Le and F. S. Ling, "Spontaneous Dissection of the Left Main Coronary Artery Treated with Percutaneous Coronary Stenting,” The Journal of Invasive Cardiology, Vol. 19, No. 8, 2007, pp. E218-E221.

[4] M. Kamran, A. Guptan and M. Bogal, "Spontaneous Coronary Artery Dissection: Case Series and Review," The Journal of Invasive Cardiology, Vol. 20, No. 10, 2008, pp. 553-559.

[5] M. Maeder, P. Ammann, W. Angehrn and H. Rickli, "Idiopathic Spontaneous Coronary Artery Dissection: Incidence, Diagnosis and Treatment," International Journal of Cardiology, Vol. 101, No. 3, 2005, pp. 363-369. doi:10.1016/j.ijcard.2004.03.045

[6] A. Hoye, "Spontaneous Coronary Artery Dissection: Time for a Concerted Effort to Better Understand This Rare Condition,” The Journal of Invasive Cardiology, Vol. 22, No. 5, 2010, pp. 229-230.

[7] S. K. Celik, A. Sagcan, A. Altintig, M. Yuksel, M. Akin and H. Kultursay, "Primary Spontaneous Coronary Artery Dissections in Atherosclerotic Patients. Report of Nine Cases with Review of the Pertinent Literature," European Journal Cardio-Thoracic Surgery, 2001, Vol. 20, No. 3, pp. 573-576. doi:10.1016/S1010-7940(01)00864-8 